\title{
Saúde Mental nos Cuidados de Saúde Primários: Desafios e Oportunidades em Contexto de Pandemia
}

\author{
Mental Health in Primary Health Care: Challenges and \\ Opportunities in the Context of a Pandemic
}

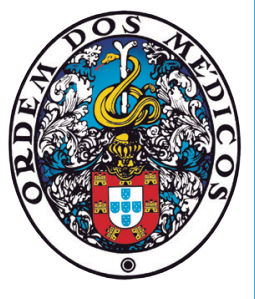

\author{
Margarida ALBUQUERQUE $\rrbracket^{* 1}$, Raquel BAPTISTA LEITE*2, Beatriz OLIVEIRA PINTO , João NUNES PIRES², \\ Vítor TRINDADE PEDROSA ${ }^{3}$, Ana Paes de VASCONCELLOS ${ }^{2}$, Joaquim GAGO ${ }^{4,5}$ \\ Acta Med Port 2021 Oct;34(10):647-649 - https://doi.org/10.20344/amp.15911
}

Palavras-chave: Ansiedade; COVID-19; Cuidados de Saúde Primários; Depressão; Indicadores Básicos de Saúde

Keywords: Anxiety; COVID-19; Depression; Health Status Indicators; Primary Health Care

\section{Indicadores básicos de saúde nos Cuidados de Saúde Primários \\ Com a reforma dos Cuidados de Saúde Primários} (CSP) de 2005, considerou-se prioritária a introdução de um processo de contratualização no Serviço Nacional de Saúde que garantisse equidade e sustentabilidade. Assim, desenvolveram-se instrumentos de medida e consideraram-se rumos de orientação, convencionalmente denominados de indicadores, que devem ter em vista a maximização dos ganhos em saúde de acordo com a mais robusta evidência científica. Existem, porém, limitações à sua utilização. Sendo fundamental que a medicina seja centrada na pessoa, os indicadores acabam por não medir aspetos primordiais como a relação médico-doente. ${ }^{1}$ Medem, no entanto, dados do processo e alguns resultados intermédios (por exemplo, valores de tensão arterial) em detrimento da avaliação final em termos de ganhos em saúde.

Para além disso, existem áreas onde não existe evidência robusta que sustente determinado indicador, como é o caso daqueles que medem a periodicidade das consultas (por exemplo o indicador 2013.054.01 «proporção de utentes com 14 ou mais anos e com o problema de 'consumo excessivo de álcool', a quem foi realizada pelo menos uma consulta relacionada nos últimos três anos») ou do pedido de meios complementares de diagnóstico. ${ }^{2}$ Apesar das limitações, estes são utilizados para a contratualização anual interna [entre os agrupamentos de centros de saúde (ACES) e as unidades funcionais] e externa (entre Administração Regional de Saúde e os ACES) dos CSP e são considerados elementos estruturantes e essenciais no novo modelo de contratualização dos CSP (2017). ${ }^{3}$

\section{Saúde Mental nos Cuidados de Saúde Primários e con- texto pandémico}

A robustez e evidência dos indicadores torna-se parti- cularmente importante em determinadas situações, como aquela sobre a qual pretendemos refletir. Dada a elevada prevalência das doenças mentais, o Plano Nacional de Saúde destaca a saúde mental como um dos principais focos de atenção dos CSP, ${ }^{4}$ que correspondem ao primeiro nível de resposta para o diagnóstico e tratamento da doença mental. No entanto, as intervenções em saúde mental não são, em geral, consideradas prioritárias nos CSP, não existindo nenhum indicador de contratualização nos CSP que avalie o impacto do seguimento em doentes com perturbação de ansiedade ou depressão.

A pandemia provocada pela COVID-19 representa um fator de risco acrescido para o desenvolvimento, exacerbação e recidiva das doenças mentais. ${ }^{5}$ Por este motivo a Organização Mundial de Saúde declara a saúde mental como um componente integrante da resposta à COVID-19. ${ }^{6}$ Durante este período, houve uma necessidade de reorganização dos serviços para atendimento a doentes COVID-19/ não-COVID-19 levando a mais desafios na prestação de cuidados aos doentes com perturbação depressiva ou ansiedade. Em abril de 2020, a Direção Geral da Saúde (DGS) publicou a norma de orientação clínica (NOC) - linhas de orientação clínica de referência em Portugal $-\mathrm{n}^{\circ}$ 011/2020 «COVID-19: Fase de mitigação - Saúde Mental», que se refere à reorganização dos serviços de saúde mental a nível hospitalar. Ainda que refira superficialmente o papel nuclear dos CSP na resposta às necessidades dos doentes com perturbações mentais ligeiras a moderadas e à relevância dos núcleos locais de resposta a catástrofe nos CSP na articulação com os serviços hospitalares de saúde mental, não especifica de que forma os doentes devem ser acompanhados, no âmbito dos CSP, durante o período da pandemia. ${ }^{7}$ A clarificação do papel dos CSP é fundamental, já que aos CSP corresponde o primeiro nível de resposta a perturbações de saúde mental, que caso

\footnotetext{
* Co-primeiros autores

1. Departamento de Saúde Mental. Hospital de Cascais. Cascais. Portugal.

2. Unidade de Saúde Familiar São Martinho de Alcabideche. Agrupamentos de Centros de Saúde de Cascais. Cascais. Portugal.

3. Unidade de Saúde Familiar KosmUS. Agrupamentos de Centros de Saúde de Cascais. Cascais. Portugal.

4. Departamento de Psiquiatria e Saúde Mental. Centro Hospitalar de Lisboa Ocidental. Lisboa. Portugal.

5. Departamento de Psiquiatria e Saúde Mental. NOVA Medical School. Lisboa. Portugal.

$\triangle$ Autor correspondente: Margarida Albuquerque. amcrs.albuquerque@gmail.com

Recebido: 18 de fevereiro de 2021 - Aceite: 24 de agosto de 2021 - Online issue published: 01 de outubro de 2021

Copyright $\odot$ Ordem dos Médicos 2021
} 
entrem em falência, levarão à sobrecarrega dos cuidados de saúde secundários (serviços de Psiquiatria), que devem ter como foco responder às doenças mentais graves. Fica assim comprometido todo o sistema de saúde mental bem como a acessibilidade, continuidade e prestação de cuidados.

Segundo a referida NOC 011/20207 o encaminhamento devido dos doentes com perturbações mentais deve seguir a boa prática clínica e os modelos já consolidados nas normas da DGS. Assim sendo, a implementação das práticas descritas na NOC nº 034/2012 «Terapêutica Farmacológica da Depressão Major e da sua Recorrência no Adulto» de 30/12/2012, ${ }^{8}$ para o tratamento da perturbação depressiva major, pressupõe a monitorização com os seguintes indicadores:

- Percentagem de pessoas com depressão major prescritas com antidepressivo;

- Percentagem de pessoas, de 55 ou mais anos, com primeiro episódio de depressão major sob terapêutica;

- Percentagem de pessoas com depressão major com segunda consulta até oito semanas após início da terapêutica.

Similarmente, a implementação da NOC no 055/2011 «Tratamento Sintomático da Ansiedade e da Insónia com Benzodiazepinas e Fármacos Análogos» ${ }^{9}$ pressupõe os indicadores:

- Proporção de utentes com o diagnóstico de ansiedade a quem foram prescritos benzodiazepinas ou fármacos análogos por períodos inferiores a $12 \mathrm{se}$ manas;

- Proporção de utentes com perturbações do sono/ insónia medicados com benzodiazepinas hipnóticas ou fármacos análogos durante menos de quatro semanas; custo médio com benzodiazepinas e fármacos análogos prescritos por utente com sintomas de ansiedade (P01) e insónia (P06) durante o período em análise.

Tanto as NOC da DGS como outras orientações clínicas enfatizam a importância de um adequado seguimento dos doentes com diagnóstico de depressão ou perturbação de ansiedade (Tabela 1), justificando esta necessidade pela

Tabela 1 - Orientações clínicas para o acompanhamento de doentes com diagnóstico de perturbação depressiva major, ansiedade ou insónia

\begin{tabular}{ll}
\hline Depressão & \\
\hline NOC $n^{\circ} 034 / 2012^{8}$ & $\begin{array}{l}\text { Na terapêutica farmacológica da pessoa com depressão major, os antidepressivos } \\
\text { são prescritos na sua fase aguda; }\end{array}$ \\
& $\begin{array}{l}\text { A consulta para avaliação da efetividade da terapêutica antidepressiva é realizada } \\
\text { no prazo máximo de oito semanas a partir da data do início da terapêutica; }\end{array}$ \\
& Se pelas 4 - 8 semanas (em média seis semanas) após o início do tratamento não \\
& se tiver observado uma melhoria pelo menos moderada dos sintomas (redução de, \\
pelo menos, $50 \%$ da intensidade dos sintomas, sendo considerado critério para & resposta ao tratamento) deve proceder-se a uma reavaliação clínica do doente, \\
com o objetivo de reconsiderar o diagnóstico, avaliar efeitos adversos, complica- & \\
ções médicas, fatores psicossociais, confirmar a adesão à medicação.
\end{tabular}

American Psychiatric Association, $2010^{8}$ - Se seis a oito semanas após o início do tratamento, o doente não tiver reduzido pelo menos $50 \%$ dos sintomas, deve ser considerada a mudança de antidepressivo para um outro com mecanismo de ação diferente, ou a reavaliação do diagnóstico, bem como a pesquisa de comorbilidades ou fatores psicossociais.

The National Institute for Health and Care Excellence, $2017^{10}$
- Se seis a oito semanas após o início do tratamento, o doente não tiver reduzido pelo menos $50 \%$ dos sintomas, deve ser considerada a adição de um segundo antidepressivo ou de um antipsicótico de segunda geração.

\begin{tabular}{|c|c|}
\hline Ansiedade & \\
\hline NOC $055 / 2011^{9}$ & $\begin{array}{l}\text { - Utilização de benzodiazepinas ansiolíticas e hipnóticas no tratamento da ansieda- } \\
\text { de ou da insónia apenas a situações de perturbação grave e incapacitante e em } \\
\text { utentes sujeitos a desgaste intenso; } \\
\text { - A duração do tratamento com estes medicamentos deve ser curta devido ao risco } \\
\text { elevado de dependência e habituação e consequências funcionais psicomotoras; } \\
\text { - Para a indicação ansiedade o tratamento não deverá ultrapassar as oito a } 12 \text { se- } \\
\text { manas e para a indicação insónia quatro semanas, estando incluído nestes perío- } \\
\text { dos, a descontinuação; } \\
\text { Quando for necessária a extensão do tratamento, o utente deverá ser reavaliado } \\
\text { em consulta especializada. }\end{array}$ \\
\hline $\begin{array}{l}\text { The National Institute for Health and } \\
\text { Care Excellence, } 2011^{11}\end{array}$ & $\begin{array}{l}\text { - Utilização de benzodiazepinas por um máximo de duas a quatro semanas; } \\
\text { Utilização de um inibidor selectivo da recaptação de serotonina como primeira } \\
\text { linha de tratamento (alternativamente pregabalina ou inibidor da recaptação de } \\
\text { serotonina/noradrenalina); intervenção psicológica baseada na terapia cognitivo- } \\
\text {-comportamental. }\end{array}$ \\
\hline
\end{tabular}


evidência científica associada ao tempo necessário para a ação da terapêutica farmacológica.

Atendendo ao impacto da pandemia na saúde mental das populações, à pressão aumentada nos CSP para dar resposta aos vários doentes e à parca referência sobre a melhor forma de seguimento destes doentes no contexto dos CSP na NOC 011/2020 «COVID-19: Fase de mitigação - Saúde Mental», ${ }^{7}$ podemos equacionar que a acessibilidade dos doentes com patologia mental possa estar comprometida.

\section{Perspetiva para o ano 2021}

Consideramos que a pandemia por COVID-19 constitui um desafio acrescido para os cuidados em saúde mental nos CSP mas também uma oportunidade para que estes sejam monitorizados. Desta forma, perante a possível redução na acessibilidade dos utentes à consulta propomos que sejam criados os indicadores relacionados com o processo (com base nas linhas orientadoras para o seguimento destes doentes), que apesar de propostos nas NOC $n^{\circ}$ 034/2012 «Terapêutica Farmacológica da Depressão Major e da sua Recorrência no Adulto», ${ }^{8}$ e n 055/2011 «Tratamento Sintomático da Ansiedade e da Insónia com Benzodiazepinas e Fármacos Análogos» ${ }^{9}$ nunca foram construídos: indicador 'Proporção de utentes com o diagnóstico de ansiedade a quem foram prescritos benzodiazepinas ou fármacos análogos por períodos inferiores a 12 semanas' e indicador 'percentagem de pessoas com depressão major com segunda consulta até oito semanas após início da terapêutica'. Também, e em linha de conta com o referido na NOC n 011/2020 «COVID-19: Fase de mitigação - Saúde Mental», ${ }^{7}$ salientamos a importância de que sejam desenhados indicadores relacionados com a estrutura (acessibilidade aos cuidados de saúde), nomeadamente através da criação de um indicador que contemple a teleconsulta como instrumento de seguimento dos doentes. Ainda no que refere à NOC $n^{\circ} 011 / 2020$ «COVID-19: Fase de

\section{REFERÊNCIAS}

1. Santos I, Ribeiro IR. Indicadores de desempenho na consulta. Rev Port Clin Geral. 2009;25:228-36.

2. Melo M, Sousa JC. Os indicadores de desempenho contratualizados com as USF: um ponto da situação no actual momento da Reforma. Rev Port Clin Geral. 2011;27:28-34.

3. Administração Central do Sistema de Saúde. Operacionalização da contratualização nos Cuidados de Saúde Primários. Biénio 2020/2021. [consultado 2021 maio 27]. Disponível em: http://www.acss.minsaude.pt/wp-content/uploads/2021/03/ACSS-Operacionalizacao_ CSP_2021_2020-VFINAL-.pdf.

4. Direção-Geral da Saúde. Plano nacional de saúde 20112016 (Vol. 2); 2017 [consultado 2020 dez 14]. Disponível em: http://1nj5ms2lli5hdggbe3mm7ms5.wpengine.netdna-cdn.com/ files/2010/08/CSH2.pdf.

5. Galea S, Merchant RM, Lurie N. The mental health consequences of COVID-19 and physical distancing - the need for prevention and early intervention. JAMA Intern Med. 2020;180:817-8.

6. World Health Organization. Maintaining essential health services: operational guidance for the COVID-19 context. Interim guidance; 2020. [consultado 2020 dez 13]. Disponível em: https://apps.who.int/iris/ mitigação - Saúde Mental», ${ }^{7}$ existe uma oportunidade para que seja atualizada tendo em conta as particularidades dos CSP, clarificando: o papel dos CSP na resposta às doenças mentais no âmbito da pandemia; como efetuar o rastreio de ansiedade e depressão no âmbito de teleconsulta; qual a estrutura de teleconsulta a adotar para o seguimento da ansiedade e depressão.

É de realçar ainda a necessidade de uma codificação regular das doenças mentais para que se possa fazer um planeamento adequado, quer do ponto de vista da prestação de cuidados aos doentes, quer do ponto de vista da governança e da saúde pública.

\section{CONTRIBUTO DOS AUTORES}

TMA, RBP: Conceptualização e desenho do artigo. Recolha e análise de dados. Redação do artigo. revisão crítica de todos os conteúdos. Elaboração e revisão de aspectos estruturais. Aprovação final do artigo.

BOP, JNP, VTP: Conceptualização e desenho do artigo. Interpretação de dados parciais. Redação do artigo. Revisão crítica de todos os conteúdos. Elaboração e revisão de aspectos estruturais. Aprovação final do artigo.

APV, JG: Conceptualização e desenho do artigo. Redação do artigo. Revisão crítica de todos os conteúdos. Elaboração e revisão de aspectos estruturais. Aprovação final do artigo.

\section{CONFLITOS DE INTERESSE}

Os autores declaram não ter conflitos de interesse relacionados com o presente trabalho.

\section{FONTES DE FINANCIAMENTO}

Este trabalho foi realizado de forma voluntária e não remunerada pelos autores, sem custos adicionais às entidades que gerem as instituições e sem prejuízo do horário de prestação de cuidados aos utentes.

handle/10665/332240

7. Direção-Geral da Saúde. Covid-19: Norma Saúde Mental - fase mitigação; 2020. [consultado 2020 dez 12]. Disponível em: https://www. dgs.pt/directrizes-da-dgs/normas-e-circulares-normativas/norma-n0112020-de-18042020-pdf.aspx.

8. Direção-Geral da Saúde. Terapêutica farmacológica da depressão major e da sua recorrência no adulto; 2012. [consultado 2020 dez 12] Disponível em: https://nocs.pt/terapeutica-depressao-major/.

9. Direção-Geral da Saúde. Tratamento sintomático da ansiedade e insónia com benzodiazepinas e fármacos análogos; 2015. [consultado 2020 dez 12]. Disponível em: https://nocs.pt/tratamento-ansiedadeinsonia-com-benzodiazepinas/.

10. The National Institute for Health and Care Excellence. Depression in adults: treatment and management. Full Guideline. (Draft for Consultation); 2017, [consultado 2020 dez 11]. Disponível em: https:// www.nice.org.uk/guidance/gid-cgwave0725/documents/draft-guideline.

11. The National Institute for Health and Care Excellence. Generalised anxiety disorder and panic disorder in adults: management. Clinical Guideline 113; 2011. [consultado 2020 dez 11]. Disponível em: http:// guidance.nice.orf.uk/CG113. 